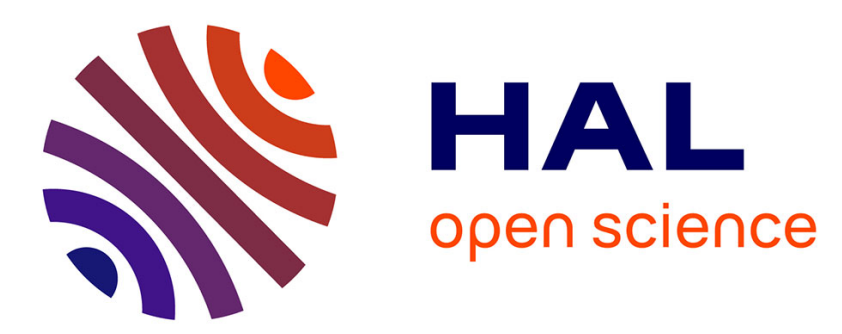

\title{
Derivation of a model of imperfect interface with finite strains and damage by asymptotic techniques: an application to masonry structures
}

\author{
Maria Letizia Raffa, Frédéric Lebon, Raffaella Rizzoni
}

\section{- To cite this version:}

Maria Letizia Raffa, Frédéric Lebon, Raffaella Rizzoni. Derivation of a model of imperfect interface with finite strains and damage by asymptotic techniques: an application to masonry structures. Meccanica, 2017, 53 (7), pp.1645-1660. 10.1007/s11012-017-0765-3 . hal-01695316

\section{HAL Id: hal-01695316 https://hal.science/hal-01695316}

Submitted on 8 Jun 2018

HAL is a multi-disciplinary open access archive for the deposit and dissemination of scientific research documents, whether they are published or not. The documents may come from teaching and research institutions in France or abroad, or from public or private research centers.
L'archive ouverte pluridisciplinaire HAL, est destinée au dépôt et à la diffusion de documents scientifiques de niveau recherche, publiés ou non, émanant des établissements d'enseignement et de recherche français ou étrangers, des laboratoires publics ou privés. 


\title{
Derivation of a model of imperfect interface with finite strains and damage by asymptotic techniques. Application to masonry structures
}

\author{
Maria Letizia Raffa • Frédéric Lebon · \\ Raffaella Rizzoni
}

Received: date / Accepted: date

\begin{abstract}
The proposed study aims to derive an imperfect interface model which couples finite strain and damaging. The governing equations are obtained via an asymptotic approach within the finite strain theory. Theoretical findings have been numerically validated within an original application to brick/mortar interfaces in masonry walls in shear loading conditions.
\end{abstract}

Keywords Bonding · Asymptotic analysis · Finite strains · Damage · Imperfect interface $\cdot$ brick/mortar interface

\section{Introduction}

In the last decades, the safeguard of ancient masonry monumental structures has animated many researchers to develop new constitutive models and innovative tools of structural analysis able to simulate the complex response of such a structure subject to static and dynamic loads.

The complexity of mechanical behavior of masonry is due to its composite nature. Masonry is an assembly of blocks of artificial or natural origin joined by dry or mortar joints. The presence of the mortar joints influences the mechanical response of the structure and provides elements of weakness. Moreover, it

M.L. Raffa

CNRS, Laboratoire de Modelisation et de Simulation Multi-Echelle, UMR CNRS 8208, France

E-mail: maria-letizia.raffa@u-pec.fr

F. Lebon

Aix-Marseille Univ., CNRS, Centrale Marseille, Laboratory of Mechanics and Acoustics,

France

E-mail: lebon@lma.cnrs-mrs.fr

R. Rizzoni

Department of Engineering, University of Ferrara, Italy

E-mail: raffaella.rizzoni@unife.it 
is well-established that brick/mortar interfaces constitute privileged zones for microcracks nucleation and propagation.

To effectively model the masonry material, two main approaches are usually adopted: a macromodelling approach which considers an equivalent fictitious material where the presence of the mortar is smeared out (e.g., $[2,27,31,30$, $43,44]$ ) and a micromodelling approach which analyzes the structure as an assembly of blocks connected by interfaces (e.g., $[13,25,26,28,50])$. The latter approach turns out to provide a more realistic description of the masonry, because it allows to adopt suitable constitutive models for each components constituting the masonry material more advantageous to the study case $[9$, $16]$.

Because of the increasing interest in describing the constitutive behavior of composite structures (i.e., fiber-reinforced materials, laminate composites, masonry, etc.), a number of interface models have been developed in the literature which take into account different interface behaviors, like elasticity, plasticity, viscoelasticity, thermal effects, residual stresses and damaging (e.g., $[3-6,8,10,14,15,17-24,33,35,39-42,45])$.

Constitutive models for interphases/interfaces in masonry structures are usually formulated in terms of the contact traction and relative displacements of the two surfaces interacting through the mortar joints $[1,9,16]$. Thus, the mortar joints are generally approximated with material interphases in which material nonlinearities eventually occur. From a numerical point of view, these constitutive laws being an internal spring-like boundary condition can be implemented within the finite element formulation via zero-thickness interface elements [15].

In this paper, a regular masonry made of blocks connected together by regular but not too thin layers of mortar is considered. The original contribution of the paper consists in the introduction of a third thin interphase interposed between bricks and mortar joints. This material interphase represents a thin layer which has the physico-chemical properties allowing the bonding between bricks and mortar joints.

A novelty with respect the existing literature about micromodelling approach for masonry structures is thus represented by the introduction of a third component which has different mechanical properties from brick and mortar and which undergoes geometrical nonlinearities (i.e., large displacements) together with material nonlinearities (i.e., damaging). To account for large displacements, the Saint Venant-Kirchhoff constitutive model is adopted, which is the simplest among nonlinear elastic material models. Damaging behavior is modeled using a procedure similar to the approach introduced by Bonetti et al. [4]: the thin interphase interposed between bricks and mortar is assumed to be a microcracked material undergoing a degradation process ruled by a pseudopotential of dissipation. The microcracked material is a generalized Kachanov-type material. In particular, within the Kachanov theory $[46,47]$, constitutive equations are obtained via the homogenization of a microcracked material characterized by $k$ families of cracks. The elastic coefficients depend on the lengths $l_{k}$ of these cracks. In the present study, only one family 
of microcracks is considered and $l$ is taken to denote their equivalent length. Moreover, the interaction between microcracks is neglected (non-interacting approximation, cf. [46]). As a result, the equivalent microcracks length $l$ represents our damage parameter. Thus, according to the Kachanov theory for microcracked media, the elasticity coefficients of the Saint Venant-Kirchhoff material are derived and they explicitly depend on the evolving damage parameter $l$.

This novel approach used to derive the imperfect interface laws coupling homogenization for microcracked media and matched asymptotic techniques is called Imperfect Interface Approach (IIA) [34-36]. Within the large displacement framework, the IIA allows to derive a nonlinear spring-like model which can be easily implemented in a finite element analysis. In analogy to former studies [15], the proposed interface model is implemented in a commercial FEM-based software via zero-thickness interface elements including damage evolution.

The present paper is organized as follows. Section 2 is devoted to the formulation of the constitutive aspects of the damaged interphase undergoing large displacements. Then, the problem of a composite body constituted of three deformable solids, a block, a thin microcracked damaging interphase and a mortar joint bonded together, is investigated in Section 3. In details, an asymptotic expansion method is applied to the elastic problem of the composite and a model of nonlinear imperfect interface for the thin interphase is derived. In such a model, surface damage effects turn out to be included in the limit as the thickness of the thin interphase vanishes. The numerical validation is detailed in Section 4. Firstly, an analytical analysis of the brick/mortar interface is carried out, in which I and II pure loading modes in the sense of fracture mechanics are investigated. Then, two finite element analyses based on the extensive experimental campaign carried out in the nineties by Vermeltfoort and Raijmakers $[48,49]$ concerning shear tests on masonry walls, are proposed. Numerical results as well as comparisons with the experimental data are discussed. Finally, conclusions are presented in Section 5.

\section{A model of interphase}

\subsection{Generalities}

In this study a regular masonry is considered, i.e., the masonry structure is made of parallelepipedic bricks linked together by regular but not too thin layers of mortar. The novelty of this approach lies in considering as a third deformable body, a thin interphase between the brick and the mortar. The physico-chemical properties of this interphase allow the bonding between the masonry principal constituents. In the following, and without loss of generality, the interphase thickness will be considered as a constant. The interphase mechanical model is detailed in the next paragraph. 


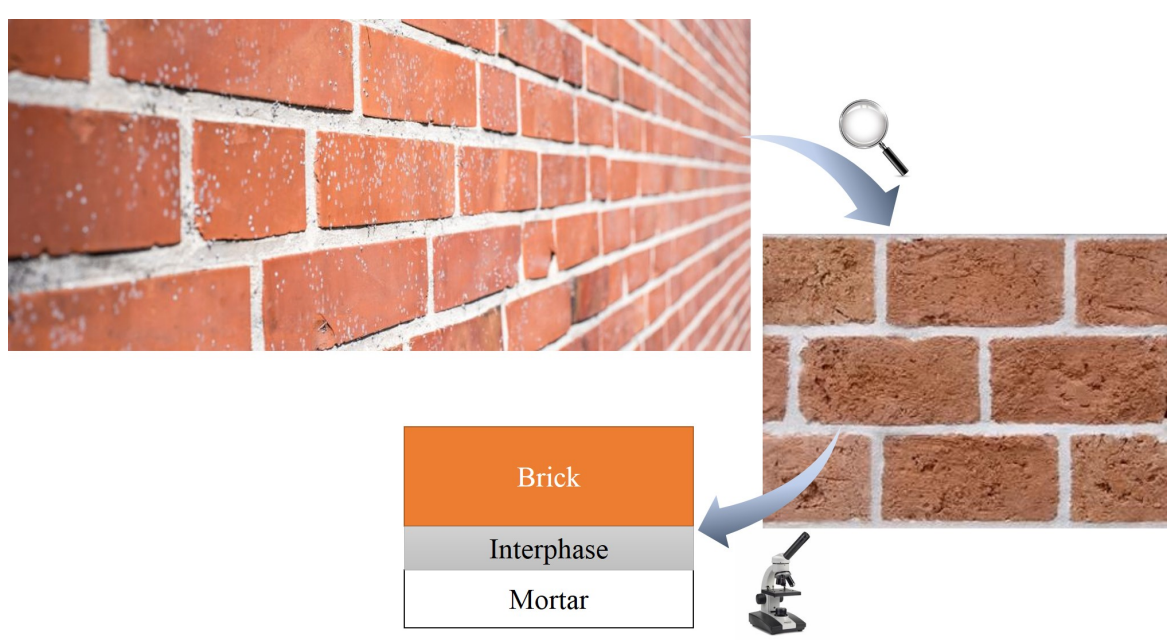

Fig. 1 Sketch of the three body modeling approach

\subsection{A model of damaged material}

In this section, a deformable solid occupying a smooth bounded domain $B$ of $\mathbb{R}^{3}$ is considered. An orthonormal Cartesian frame $\left(O, \mathbf{e}_{1}, \mathbf{e}_{2}, \mathbf{e}_{3}\right)$ is introduced and let $\left(x_{1}, x_{2}, x_{3}\right)$ be taken to denote the three coordinates of a particle. The solid is assumed to comprise a material:

- hyperelastic of a Saint Venant-Kirchhoff-type [41,35]

- microcracked of a generalized Kachanov-type [29,47]

As a result, the elastic coefficients depend on the length $l$, which is taken to denote the equivalent cracks length, therefore, it has the meaning of a damage parameter.

As an example, for an isotropic material weakened by a cracks family lying orthogonal to $\mathbf{e}_{3}$, the Young modulus in the third direction $E_{3}$ is equal to

$$
E_{3}=\frac{E_{0}}{1+2 \rho C E_{0}},
$$

with

$$
C=\frac{\pi}{2} \frac{1}{\sqrt{E_{0}}}\left(\frac{1}{\mu_{0}}-2 \frac{\nu_{0}}{E_{0}}+\frac{2}{E_{0}}\right)^{1 / 2}
$$

where $E_{0}$ (resp. $\mu_{0}$, resp. $\nu_{0}$ ) is the Young modulus (resp. the shear modulus, resp. the Poisson ratio) of the undamaged material, and $\rho$ is the cracks density, which form is $\rho=\frac{l^{3}}{V}$ in 3D problems and $\rho=\frac{l^{2}}{S}$ in 2D, being $V$ (resp. $S$ ) the volume (resp. the surface) of the representative elementary domain $[29,47]$.

Let the evolution of crack length $l$ be introduced. A pseudo-potential of dissipation $\phi$ is considered [12]. As proposed in former papers [4,32], the chosen 
pseudo-potential is given by the sum of a quadratic term and a positively 1homogeneous functional, as a result the dissipative character will be directly related to a rate-dependent and a rate-independent contributions:

$$
\phi(\dot{l})=\frac{1}{2} \eta \dot{l}^{2}+I_{[0,+\infty[}(\dot{l}),
$$

where $\eta$ is a positive viscosity parameter and $I_{A}$ denotes the indicator function of the set $A$, i.e., $I_{A}(x)=0$ if $x \in A$ and $I_{A}(x)=+\infty$ otherwise. The term $I_{[0,+\infty}[i)$ forces $i$ to assume non-negative values (i.e., the crack length cannot decrease) and it renders the irreversible character of the degradation process of the bond. Moreover, the free energy of the solid is chosen as follows:

$$
\psi(E, l)=\frac{1}{2} \mathbb{B}(l) E(u): E(u)-\omega l+I_{\left[l_{0}, \infty[\right.}(l)
$$

where $E(u)$ is the Green-Lagrange tensor, $E(u)=\frac{1}{2}\left(\nabla u+\nabla u^{T}+\nabla u^{T} \nabla u\right)$, $\mathbb{B}(l)$ is the stiffness tensor of the body, with usual properties of symmetry and positivity, $\omega$ is a (negative) parameter similar to the Dupré's energy $[12,11]$ and $l_{0}$ is a given initial crack length. It is worth to highlight that, due to the weak thickness of the bond, it seems natural to introduce finite strains in the interphase. The constitutive equation is given by:

$$
S=F^{-1} P=\mathbb{B}(l) E(u)
$$

where $P$ (resp. $S$ ) is the first (resp. second) Piola stress tensor and $F=I+\nabla u$ is the gradient of transformation tensor.

Remark. It is possible to limit the crack length by assuming a vanishing stiffness if $l \leq l_{\text {max }}$; this can be done by introducing the characteristic function of $\left[l_{0}, l_{\max }\right], \chi_{\left[l_{0}, l_{\max }\right]}$, and by assuming for the free energy the following form: $\psi(E, l)=\chi_{\left[l_{0}, l_{\max }\right]}\left(\frac{1}{2} \mathbb{B}(l) E(u): E(u)-\omega l\right)+I_{\left[l_{0}, \infty[\right.}(l)$.

Therefore, the equilibrium of the body is written as:

$$
\operatorname{Div} P+f=0, \text { in } B
$$

where $f$ is a given body force.

Finally, from the chosen form of the pseudo-potential of dissipation $\phi$ (Eq. (3)) and because of the positivity of the parameter $\eta>0$, the following equation governing the damage parameter $l$ results:

$$
\eta \dot{l}=\left(\omega-\frac{1}{2} \mathbb{B}_{, l}(l) E\left(u^{\varepsilon}\right): E(u)\right)_{+},
$$

where $(\cdot)_{+}$is taken to denote the positive part of a function. 
2.3 A model of rescaled damaged interphase

The thin interphase domain $B^{\varepsilon}$ is assumed to have a constant thickness $\varepsilon$ in the third direction $\mathbf{e}_{3}$ (see Fig. 2), i.e.

$$
B^{\varepsilon}=\left\{x \in \mathbb{R}^{3},\left|x_{3}\right|<\varepsilon / 2\right\} .
$$

In the following, all the parameters and fields are indexed by $\varepsilon$.

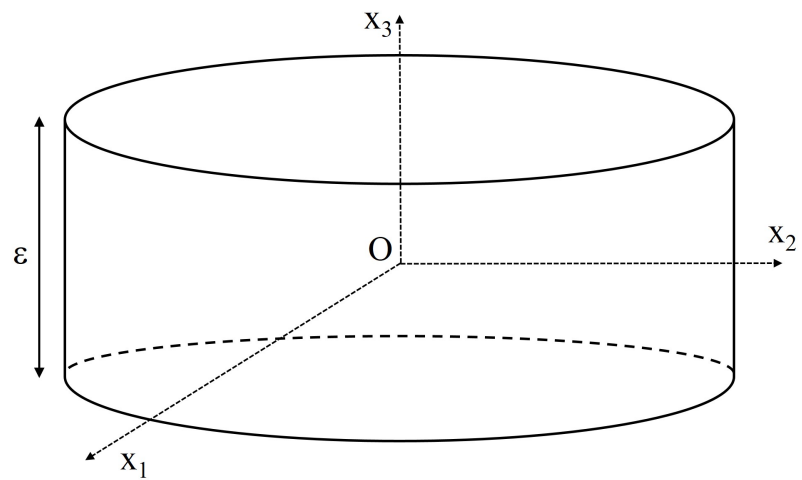

Fig. 2 An example of the geometry of the interphase

The upper and lower surfaces of the thin interphase are denoted as

$$
S_{ \pm}^{\varepsilon}=\left\{x \in \mathbb{R}^{3}, x_{3}= \pm \varepsilon / 2\right\} \quad \text { and } \quad S_{L}^{\varepsilon}=\partial B^{\varepsilon} / S_{ \pm}^{\varepsilon},
$$

where $\partial B^{\varepsilon}$ indicates the boundary of $B^{\varepsilon}$. Body forces in $B^{\varepsilon}$ and lateral forces on $S_{L}^{\varepsilon}$ are neglected. Moreover, the material body is assumed to be soft, i.e., $\mathbb{B}_{i j h k}(l) \approx \varepsilon b_{i j h k}(l)$.

Thus, the equilibrium problem of the body is described by the following system of equations:

$$
\begin{cases}P_{i j, j}^{\varepsilon}=0 & \text { in } B^{\varepsilon} \\ P_{i j}^{\varepsilon} n_{j}=0 & \text { on } S_{L}^{\varepsilon} \\ P_{i j}^{\varepsilon} n_{j}=P_{i}^{ \pm} & \text {on } S_{ \pm}^{\varepsilon} \\ P_{i j}^{\varepsilon}=\varepsilon F_{i r}^{\varepsilon} b_{r j h k}(l) E_{h k}\left(u^{\varepsilon}\right) & \text { in } B^{\varepsilon} \\ \eta^{\varepsilon} i=\left(\omega^{\varepsilon}-\frac{1}{2} \varepsilon b_{, l}(l) E\left(u^{\varepsilon}\right): E\left(u^{\varepsilon}\right)\right)_{+} & \text {in } B^{\varepsilon}\end{cases}
$$

where $P^{+}=P^{-}$are a priori given vectors.

Since the weak thickness of the interphase, it is natural to seek the solution of problem (61) using asymptotic expansions with respect to the small parameter $\varepsilon[20-24,42]$. In particular, the following asymptotic series with fractional 
powers are assumed $[41,35]$ :

$$
\left\{\begin{array}{l}
u^{\varepsilon}=u^{0}+\varepsilon^{1 / 3} u^{1}+\varepsilon^{2 / 3} u^{2}+\varepsilon u^{3}+o(\varepsilon) \\
P^{\varepsilon}=P^{0}+\varepsilon^{1 / 3} P^{1}+\varepsilon^{2 / 3} P^{2}+\varepsilon P^{3}+o(\varepsilon)
\end{array}\right.
$$

The following change of variable is introduced [7]:

$$
\left(x_{1}, x_{2}, x_{3}\right) \in B^{\varepsilon} \rightarrow\left(z_{1}, z_{2}, z_{3}\right) \in B, \text { with }\left(z_{1}, z_{2}, z_{3}\right)=\left(x_{1}, x_{2}, \frac{x_{3}}{\varepsilon}\right)
$$

and it is set $\hat{u}^{\varepsilon}\left(z_{1}, z_{2}, z_{3}\right)=u^{\varepsilon}\left(x_{1}, x_{2}, x_{3}\right)$ and $\hat{P}^{\varepsilon}\left(z_{1}, z_{2}, z_{3}\right)=P^{\varepsilon}\left(x_{1}, x_{2}, x_{3}\right)$, where $B=\left\{\left(x_{1}, x_{2}, x_{3}\right) \in \Omega:\left|x_{3}\right|<\frac{1}{2}\right\}$. The superscript $(\wedge)$ denotes the fields in the rescaled configuration.

The governing equations of the rescaled problem result in:

$$
\begin{cases}\hat{P}_{i j, j}^{\varepsilon}=0 & \text { in } B \\ \hat{P}_{i j}^{\varepsilon} n_{j}=0 & \text { on } S_{L} \\ \hat{P}_{i j}^{\varepsilon} n_{j}=\hat{P}_{i}^{ \pm} & \text {on } S_{ \pm} \\ \hat{P}_{i j}^{\varepsilon}=\varepsilon \hat{F}_{i r}^{\varepsilon} \hat{b}_{r j h k}(l) \hat{E}_{h k}\left(\hat{u}^{\varepsilon}\right) & \text { in } B \\ \hat{\eta}^{\varepsilon} \dot{\hat{l}}=\left(\hat{\omega}^{\varepsilon}-\frac{1}{2} \varepsilon \hat{b}_{, l}(l) \hat{E}\left(\hat{u}^{\varepsilon}\right): \hat{E}\left(\hat{u}^{\varepsilon}\right)\right)_{+} & \text {in } B\end{cases}
$$

where $S_{ \pm}=\left\{\left(x_{1}, x_{2}, x_{3}\right) \in \Omega: x_{3}= \pm \frac{1}{2}\right\}$ and $S_{L}=\partial B / S_{ \pm}, \partial B$ are the boundaries of $B$. In Eqs. $(12), \hat{E}\left(\hat{u}^{\varepsilon}\right)$ is the rescaled Green-Lagrange tensor, and its asymptotic expansion reads as:

$$
\hat{E}\left(\hat{u}^{\varepsilon}\right)=\varepsilon^{-2} \hat{E}^{-2}+\varepsilon^{-1} \hat{E}^{-1}+\hat{E}^{0}+O(\varepsilon),
$$

where

$$
\left\{\begin{array}{l}
\hat{E}^{-2}=\frac{1}{2}\left|\hat{u}_{, 3}^{\varepsilon}\right|^{2} e_{3} \otimes e_{3}, \\
\hat{E}^{-1}=\frac{1}{2} u_{, 3}^{\varepsilon} \otimes e_{3}+\frac{1}{2}\left(u_{, 3}^{\varepsilon} \cdot u_{, \alpha}^{\varepsilon}\right)\left(e_{\alpha} \otimes e_{3}\right), \\
\hat{E}^{0}=\frac{1}{2}\left(u_{, \alpha}^{\varepsilon} \otimes e_{\alpha}\right)+\frac{1}{2} u_{3, \alpha}^{\varepsilon}\left(e_{\alpha} \otimes e_{3}+e_{3} \otimes e_{\alpha}\right)+\frac{1}{2}\left(u_{, \alpha}^{\varepsilon} \cdot u_{, \beta}^{\varepsilon}\right)\left(e_{\alpha} \otimes e_{\beta}\right) .
\end{array}\right.
$$

In view of Eqs. (11) the displacement and stress fields are written as asymptotic expansions in the rescaled adhesive, as follows:

$$
\left\{\begin{array}{l}
\hat{P}^{\varepsilon}=\hat{P}^{0}+\varepsilon^{1 / 3} \hat{P}^{1}+\varepsilon^{2 / 3} \hat{P}^{2}+\varepsilon \hat{P}^{1}+o(\varepsilon) \\
\hat{u}^{\varepsilon}=\hat{u}^{0}+\varepsilon^{1 / 3} \hat{u}^{1}+\varepsilon^{2 / 3} \hat{u}^{2}+\varepsilon \hat{u}^{1}+o(\varepsilon) .
\end{array}\right.
$$

Substituting Eqs. (15) into the first equation of system (12), the following conditions are found to hold in $B$ :

$$
\hat{P}_{i 3,3}^{k}=0, k=0,1,2, i=1,2,3
$$

The latter result implies that $\hat{P}_{i 3}^{k}$ is independent of $z_{3}$ and, additionally, that the relative jumps are equal to zero:

$$
\left[\hat{P}_{i 3}^{k}\right]=0, k=0,1,2, i=1,2,3
$$


where the notation $[f]=f\left(x_{1}, x_{2}, \frac{1}{2}\right)-f\left(x_{1}, x_{2},-\frac{1}{2}\right)$ indicates the jumps of function $f$ in the rescaled model.

Substituting Eqs. (15) and Eqs. (14) into the fourth equation of system (12) it is deduced that the following conditions hold in $B$ :

$$
\hat{u}_{i, 3}^{k}=0, k=0,1, \quad i=1,2,3
$$

and

$$
\hat{u}_{i, 33}^{2}=0, i=1,2,3
$$

i.e. $\hat{u}_{i}^{k}$ and $\hat{u}_{i, 3}^{2}$ are independent of $z_{3}$, leading to the conditions:

$$
\begin{gathered}
{\left[\hat{u}^{k}\right]=0, k=0,1,} \\
\hat{u}_{, 3}^{2}=\left[\hat{u}^{2}\right] .
\end{gathered}
$$

Moreover, the following relationship is obtained:

$$
\left(\frac{1}{2} \hat{b}_{3333}(l)\left|\hat{u}_{i, 3}^{2}\right|^{2} \hat{u}_{i, 3}^{2}\right)_{, 3}=0
$$

and thus

$$
\frac{1}{2} \hat{b}_{3333}(l)\left|\hat{u}_{i, 3}^{2}\right|^{2} \hat{u}_{i, 3}^{2}=\hat{P}^{0}
$$

where $\hat{P}^{0}$ is the first term in the expansion of $\hat{P}$. By introducing Eq. (21) in Eq. (23) the following equation is obtained:

$$
\hat{P}^{0}=\frac{1}{2} \hat{b}_{3333}(l)\left|\left[\hat{u}^{2}\right]\right|^{2}\left[\hat{u}^{2}\right]
$$

Let consider the fifth equation in system (12). The term

$$
\frac{1}{2} \varepsilon \int_{-\frac{1}{2}}^{\frac{1}{2}} \hat{b}_{, l}(l) \hat{E}\left(\hat{u}^{\varepsilon}\right): \hat{E}\left(\hat{u}^{\varepsilon}\right) d z_{3}
$$

has to be estimated. Using the asymptotic expansions of $\hat{u}^{\varepsilon}$ and Eqs. (18)-(19), the first non-vanishing term in the expansion (the second order term) is found to be governed by the expansion of $\hat{E}_{33}\left(\hat{u}^{\varepsilon}\right)$. Eqs. (18) and (19) leads to

$$
\hat{E}_{33}\left(\hat{u}^{\varepsilon}\right) \approx \frac{1}{2} \varepsilon^{-2 / 3}\left(\hat{u}_{1,3}^{2}+\hat{u}_{2,3}^{2}+\hat{u}_{3,3}^{2}\right)
$$

As a results, Eq. (25) can be rewritten as follows:

$$
\begin{aligned}
& \frac{1}{2} \varepsilon \int_{-\frac{1}{2}}^{\frac{1}{2}} \hat{b}_{, l}(l) \hat{E}\left(\hat{u}^{\varepsilon}\right): \hat{E}\left(\hat{u}^{\varepsilon}\right) d z_{3} \approx \\
& \approx \frac{1}{2} \hat{b}_{3333, l}(l) \int_{-\frac{1}{2}}^{\frac{1}{2}}\left(\frac{1}{2} \varepsilon^{-2 / 3}\left(\left(\hat{u}_{1,3}^{2}\right)^{2}+\left(\hat{u}_{2,3}^{2}\right)^{2}+\left(\hat{u}_{3,3}^{2}\right)^{2}\right)\right)^{2} d z_{3}
\end{aligned}
$$


It is worth recalling that the second order terms in the displacements expansion $\hat{u}_{i, 3}^{2}, i=1,2,3$ are found to be constant with respect to $z_{3}$ (cf. Eq. (19)), accordingly, the expression $\left(\hat{u}_{1,3}^{2}\right)^{2}+\left(\hat{u}_{2,3}^{2}\right)^{2}+\left(\hat{u}_{3,3}^{2}\right)^{2}$ leads to $\left|\left[\hat{u}^{2}\right]\right|^{2}$ after integration.

Finally, the damage evolution equation results in:

$$
\hat{\eta}^{-1} \dot{\hat{l}}=\left(\hat{\omega}^{-1}-\frac{1}{8} \hat{b}_{3333, l}(l)\left|\left[\hat{u}^{2}\right]\right|^{4}\right)_{+}
$$

where $\eta^{-1}$ and $\omega^{-1}$ are the terms in the expansion of $\eta^{\varepsilon}$ and $\omega^{\varepsilon}$ respectively of power $-1 / 3$,

$$
\left\{\begin{array}{l}
\hat{\eta}^{\varepsilon}=\ldots+\varepsilon^{-1 / 3} \hat{\eta}^{-1}+\ldots \\
\hat{\omega}^{\varepsilon}=\ldots+\varepsilon^{-1 / 3} \hat{\omega}^{-1}+\ldots
\end{array}\right.
$$

One remarks that $\eta^{\varepsilon}$ and $\omega^{\varepsilon}$ are assumed to be independent of the $z_{3}$ coordinate.

\section{Study of a composite}

The body $B^{\varepsilon}$ studied in the two previous sections is, in what follows, sandwiched between two hyperelastic bodies occupying respectively the domains $\Omega_{ \pm}^{\varepsilon} \subset \mathbb{R}^{3}$, denoted as adherents. It is assumed that $\Omega_{ \pm}^{\varepsilon}$ and $B^{\varepsilon}$ are perfectly bonded along the surfaces $S_{ \pm}^{\varepsilon}$. We assume that an external load $g$ is applied on a boundary part $S_{g}^{ \pm} \in \partial \Omega_{ \pm}^{\varepsilon} / S_{ \pm}^{\varepsilon}$, and that the boundary condition $u=0$ holds on $S_{u}^{ \pm} \in \partial \Omega_{ \pm}^{\varepsilon} / S_{ \pm}^{\varepsilon}$, with $S_{u}^{ \pm}$having strictly positive measure and such that $S_{g}^{ \pm} \cap S_{u}^{ \pm}=\emptyset$. Finally, a body force $f^{ \pm}$is applied to $\Omega_{ \pm}^{\varepsilon}$.

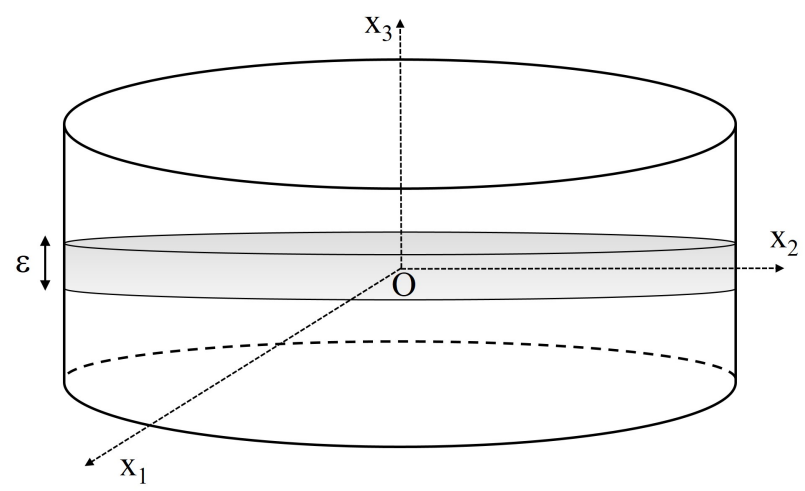

Fig. 3 An example of the geometry of the composite 
The equilibrium problem of the composite structure is described by the following system of equations:

$$
\begin{cases}P_{i j, j}^{\varepsilon}+f_{i}^{ \pm}=0 & \text { in } \Omega_{ \pm}^{\varepsilon} \\ P_{i j}^{\varepsilon} n_{j}=g_{i}^{ \pm} & \text {on } S_{g} \\ P_{i j, j}^{\varepsilon}=0 & \text { in } B^{\varepsilon} \\ {\left[\left[P_{i 3}^{\varepsilon}\right]\right]=0} & \text { on } S_{ \pm}^{\varepsilon} \\ {\left[\left[u_{i}^{\varepsilon}\right]\right]=0} & \text { on } S_{ \pm}^{\varepsilon} \\ u_{i}^{\varepsilon}=0 & \text { on } S_{u}^{ \pm} \\ S_{i j}^{\varepsilon}=a_{i j h k}^{ \pm} E_{h k}\left(u^{\varepsilon}\right) & \text { in } \Omega_{ \pm}^{\varepsilon} \\ S_{i j}^{\varepsilon}=\varepsilon b_{i j h k}(l) E_{h k}\left(u^{\varepsilon}\right) & \text { in } B^{\varepsilon} \\ \eta^{\varepsilon} i=\left(\omega^{\varepsilon}-\frac{1}{2} \varepsilon b_{, l}^{\varepsilon}(l) E\left(u^{\varepsilon}\right): E\left(u^{\varepsilon}\right)\right)_{+} & \text {in } B^{\varepsilon}\end{cases}
$$

where $a^{ \pm}$are the elasticity tensors in $\Omega_{ \pm}^{\varepsilon}$.

The following change of variable is introduced in the adherents: $\left(x_{1}, x_{2}, x_{3}\right) \in$ $\Omega_{ \pm}^{\varepsilon} \rightarrow\left(z_{1}, z_{2}, z_{3}\right) \in \Omega_{ \pm}$, with $\left(z_{1}, z_{2}, z_{3}\right)=\left(x_{1}, x_{2}, x_{3} \pm 1 / 2 \mp \varepsilon / 2\right)$, and it is set $\bar{u}^{\varepsilon}\left(z_{1}, z_{2}, z_{3}\right)=u^{\varepsilon}\left(x_{1}, x_{2}, x_{3}\right)$ and $\bar{P}^{\varepsilon}\left(z_{1}, z_{2}, z_{3}\right)=P^{\varepsilon}\left(x_{1}, x_{2}, x_{3}\right)$, where $\Omega_{ \pm}=\left\{\left(x_{1}, x_{2}, x_{3}\right) \in \Omega: \pm x_{3}>\frac{1}{2}\right\}$. The external forces are assumed to be independent of $\varepsilon$. As a consequence, it is set $\bar{f}^{ \pm}\left(z_{1}, z_{2}, z_{3}\right)=f^{ \pm}\left(x_{1}, x_{2}, x_{3}\right)$ and $\bar{g}^{ \pm}\left(z_{1}, z_{2}, z_{3}\right)=g^{ \pm}\left(x_{1}, x_{2}, x_{3}\right)$. Note that the superscript - (respectively $\wedge$ ) denotes the fields in the adherents $\Omega_{ \pm}^{\varepsilon}$ (respectively in the interphase $B^{\varepsilon}$ ) in the rescaled configuration.

The governing equations of the rescaled problem are the following:

$$
\begin{cases}\bar{P}_{i j, j}^{\varepsilon}+\bar{f}_{i}^{ \pm}=0 & \text { in } \Omega_{ \pm} \\ \bar{P}_{i j}^{\varepsilon} n_{j}=\bar{g}_{i}^{ \pm} & \text {on } \bar{S}_{g}^{ \pm} \\ \hat{P}_{i j, j}^{\varepsilon}=0 & \text { in } B \\ \bar{P}_{i 3}^{\varepsilon}=\hat{P}_{i 3}^{\varepsilon} & \text { on } S_{ \pm} \\ \bar{u}_{i}^{\varepsilon}=\hat{u}_{i}^{\varepsilon} & \text { on } S_{ \pm} \\ \bar{u}_{i}^{\varepsilon}=0 & \text { on } \bar{S}_{u} \\ \bar{S}_{i j}^{\varepsilon}=\bar{a}_{i j h k}^{ \pm} \bar{E}_{h k}\left(\bar{u}^{\varepsilon}\right) & \text { in } \Omega_{ \pm} \\ \hat{S}_{i j}^{\varepsilon}=\varepsilon \hat{b}_{i j h k}(l) \hat{E}_{h k}\left(\hat{u}^{\varepsilon}\right) & \text { in } B \\ \hat{\eta}^{\varepsilon} \dot{\hat{l}}^{\varepsilon}\left(\hat{\omega}^{\varepsilon}-\frac{1}{2} \varepsilon \hat{b}_{, l}(l) \hat{E}\left(\hat{u}^{\varepsilon}\right): \hat{E}\left(\hat{u}^{\varepsilon}\right)\right)_{+} & \text {in } B\end{cases}
$$

In view of Eqs. (11), the displacement and stress fields, written as asymptotic expansions, read as:

$$
\left\{\begin{array}{l}
\bar{P}^{\varepsilon}=\bar{P}^{0}+\varepsilon^{1 / 3} \bar{P}^{1}+\varepsilon^{2 / 3} \bar{P}^{2}+\varepsilon \bar{P}^{3}+o(\varepsilon) \\
\bar{u}^{\varepsilon}=\bar{u}^{0}+\varepsilon^{1 / 3} \bar{u}^{1}+\varepsilon^{2 / 3} \bar{u}^{2}+\varepsilon \bar{u}^{3}+o(\varepsilon),
\end{array}\right.
$$

Substituting Eqs. (32) in system (31), the first order of expansions (power $0)$ provides the following conditions: 


$$
\begin{cases}\bar{P}_{i j, j}^{0}+\bar{f}_{i}^{ \pm}=0 & \text { in } \Omega_{ \pm} \\ \bar{P}_{i j}^{0} n_{j}=\bar{g}_{i}^{ \pm} & \text {on } \bar{S}_{g}^{ \pm} \\ \bar{u}_{i}^{0}=0 & \text { on } \bar{S}_{u}^{ \pm} \\ \bar{S}_{i j}^{0}=\bar{a}_{i j h k}^{ \pm} \bar{E}_{h k}\left(\bar{u}^{0}\right) & \text { in } \Omega_{ \pm}\end{cases}
$$

Using the results obtained in Section 2, we have that the following conditions

$$
\bar{P}_{i 3}^{0}=\frac{1}{2} \hat{b}_{3333}(l)\left|\left[\hat{u}^{2}\right]\right|^{2}\left[\hat{u}_{i}^{2}\right]
$$

and

$$
\hat{\eta}^{-1} \dot{\hat{l}}=\left(\hat{\omega}^{-1}-\frac{1}{8} b_{3333, l}(l)\left|\left[\hat{u}^{2}\right]\right|^{4}\right)_{+}
$$

hold on the surfaces $S_{ \pm}$. The latter conditions are complemented by the continuity conditions of the stress vectors along the surfaces $S_{ \pm}$.

To summarize, the following system of equations is obtained:

$$
\begin{cases}P_{i j, j}+f_{i}^{ \pm}=0 & \text { in } \Omega_{ \pm} \\ P_{i j} n_{j}=g_{i}^{ \pm} & \text {on } \bar{S}_{g}^{ \pm} \\ u_{i}=0 & \text { on } \bar{S}_{u}^{ \pm} \\ S_{i j}=a_{i j h k}^{ \pm} E_{h k}(u) & \text { in } \Omega_{ \pm} \\ {\left[P_{i 3}\right]=0} & \text { on } S \\ P_{i 3}=\frac{1}{2 \varepsilon^{2}} b_{3333}(l)|[u]|^{2}\left[u_{i}\right] & \text { on } S \\ \eta \dot{l}=\left(\omega-\frac{1}{8 \varepsilon^{3}} b_{3333, l}(l)|[u]|^{4}\right)_{+} & \text {on } S\end{cases}
$$

The system (36) governs the equilibrium problem of an adhesive interface characterized by a nonlinear damaging behavior, which is sandwiched between two adherents. In the next section, some numerical and analytical applications of the proposed interface model concerning modeling of the brick/mortar interfaces in masonry structures are investigated.

\section{Numerical validations}

4.1 Analytical analysis of the brick/mortar interface model

In what follows, two simple analytical applications of the proposed soft interface model are presented. The presence of I and II pure loading modes in the sense of fracture mechanics is investigated. The geometrical configurations are shown in Fig. 4. 

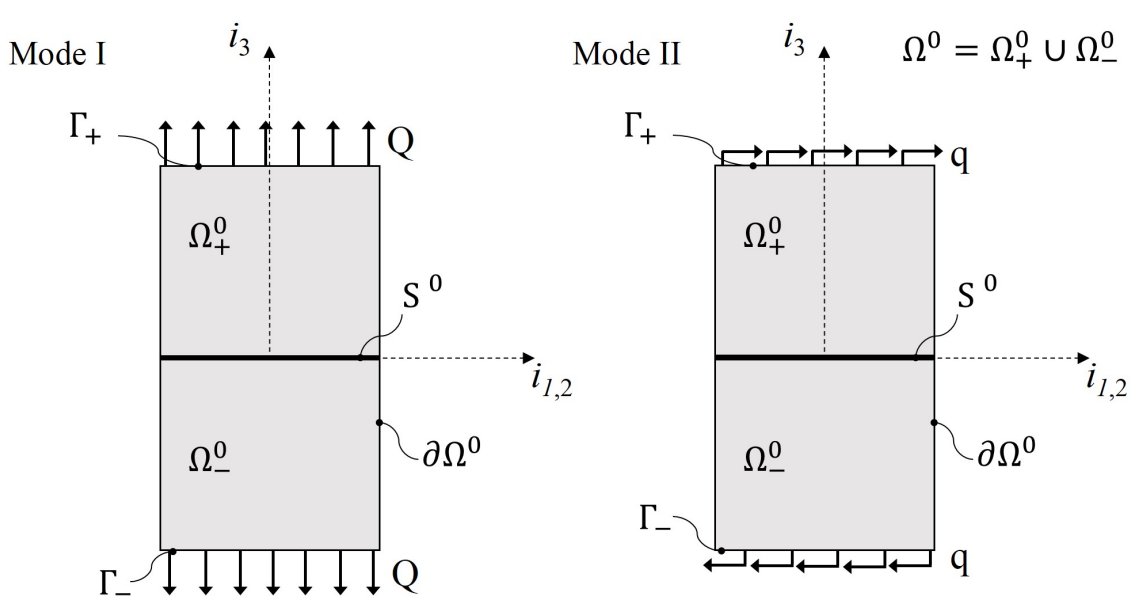

Fig. 4 Sketch of the investigated systems composed of two nonlinear elastic isotropic adherents $\Omega_{+}^{0}$ and $\Omega_{-}^{0}$ joined by a nonlinear soft interface condition along a common face $S^{0}$. I and II pure loading modes are represented on the left and on the right side, respectively

\subsubsection{Mode I: uniaxial tension/compression condition}

The proposed example is based on the former paper by R. Rizzoni et al. [41] (Section 7). In the reference configuration, the composite structure is subjected to a tensile (compressive load) $Q>0(Q<0)$ directed along $i_{3}$ and acting on the upper and the lower boundaries $\Gamma_{+}$and $\Gamma_{-}$, as shown in Fig. 4. On the remaining part of the boundary $\partial \Omega^{0}$, the surface forces are taken to vanish. The load intensity $Q$ is assumed to not depend on $\varepsilon$ and the body forces are neglected. For the sake of simplicity, the adherents are supposed to comprise the same Saint Venant-Kirchhoff material with Lamé constants $\lambda$ and $\mu$, however the engineering elastic constants $E$ and $\nu$ are used in what follows.

By combining the formulation proposed in [41] with the one obtained in Sections 2-3 (cf. Eqs. (36)), the equilibrium problem of the joined structure reads as:

$$
\begin{cases}\operatorname{Div} P=0 & \text { in } \Omega^{0} \\ P=(I+\nabla u)\left[\left(\frac{E}{1+\nu}\right) E(u)+\frac{\nu E}{(1+\nu)(1-2 \nu)}(I \cdot E(u)) I\right] & \text { in } \Omega^{0} \\ E(u)=\frac{1}{2}\left(\nabla u+\nabla u^{T}+\nabla u^{T} \nabla u\right) & \text { in } \Omega^{0} \\ {\left[P i_{3}\right]=0} & \text { on } S^{0} \\ P i_{3}=\frac{1}{2 \varepsilon^{2}} b_{3333}(l)|[u]|^{2}[u] & \text { on } S^{0} \\ P i_{\alpha}=0 \quad \text { with } \alpha=1,2 & \text { on } \partial \Omega^{0} \\ P i_{3}=Q i_{3} & \text { on } \Gamma_{ \pm} \\ \eta i=\left(\omega-\frac{1}{8 \varepsilon^{3}} b_{3333, l}(l)|[u]|^{4}\right)_{+} & \text {on } S^{0}\end{cases}
$$


We seek the following solution for the displacement field $u: \Omega^{0} \rightarrow \mathbb{R}^{3}$ :

$$
u=\left(\lambda_{1}-1\right)\left(x_{1} i_{1}+x_{2} i_{2}\right)+\left(\left(\lambda_{3}-1\right) x_{3} \pm \frac{1}{2}\left[u_{3}\right]\right) i_{3} \quad \text { in } \Omega_{ \pm}^{0}
$$

with $\lambda_{1}, \lambda_{3}$ constants to be determined representing the stretches along $x_{1}$ and $x_{3}$ axes, respectively, and $\left[u_{3}\right]$ the $i_{3}$-component of the constant vector $[u]$ representing the displacement jump at the interface $S^{0}$.

The first Piola-Kirchhoff stress tensor corresponding to (38) is:

$$
P=P_{11}\left(i_{1} \otimes i_{1}+i_{2} \otimes i_{2}\right)+P_{33}\left(i_{3} \otimes i_{3}\right)
$$

with

$$
\begin{aligned}
P_{11} & =\frac{E \lambda_{1}}{2(1+\nu)(1-2 \nu)}\left(\lambda_{1}^{2}-1\right)+\frac{\nu E \lambda_{1}}{2(1+\nu)(1-2 \nu)}\left(\lambda_{3}^{2}-1\right) \\
P_{33} & =\frac{\nu E \lambda_{3}}{(1+\nu)(1-2 \nu)}\left(\lambda_{1}^{2}-1\right)+\frac{E(1-\nu) \lambda_{3}}{2(1+\nu)(1-2 \nu)}\left(\lambda_{3}^{2}-1\right)
\end{aligned}
$$

The stretch $\lambda_{1}$, obtained by imposing the vanishing of $P_{11}$ has the following form (cf. [41]):

$$
\lambda_{1}=\left(1+\nu-\nu \lambda_{3}^{2}\right)^{\frac{1}{2}}
$$

By substituting Eq. (42) in Eq. (41) and coupling the boundary condition on $\Gamma_{ \pm}$(cf. Eqs. (37)), the equation which determines $\lambda_{3}$ as a function of the load $Q$ is obtained:

$$
Q=2 E \lambda_{3}\left(\lambda_{3}^{2}-1\right)
$$

Moreover, by recasting the fifth equation of the system (37) and taking into account the boundary condition on $\Gamma_{ \pm}$, the displacement jump $\left[u_{3}\right]$ is derived:

$$
\left[u_{3}\right]=\varepsilon^{\frac{2}{3}} \frac{Q}{|Q|^{\frac{2}{3}}}\left(\frac{2}{b_{3333}(l)}\right)^{\frac{1}{3}}
$$

which is positive if $Q>0$ and negative otherwise.

In case of compression $(Q<0), \omega$ is a negative-valued constant and therefore the last equation of the system (37) gives $\eta \dot{l}=0$, implying no damage evolution. We thus consider the case of traction $(Q>0)$. After assuming $b_{3333}(l)=\frac{L}{2 C l^{2}}$, we have $b_{3333, l}(l)=-\frac{L}{C l^{3}}$, in which $C$ is a positive-defined elastic constant (see Eq. (2)) and $L$ is a constant playing the role of a characteristic interface length. By using this last expression as well as Eq. (44) in the last equation of the system (37), the damage evolution law as a function of the load $Q$ is obtained:

$$
\eta l_{, Q} \dot{Q}=\left(\omega-\left(\frac{C}{2 \varepsilon L l(Q)}\right)^{\frac{1}{3}} Q^{\frac{4}{3}}\right)_{+}
$$

Because $\omega<0$, we find no damage evolution for $0 \leq Q \leq Q_{0}$ with:

$$
Q_{0}=-\omega^{\frac{4}{3}}\left(\frac{2 \varepsilon L l_{0}}{C}\right)^{\frac{1}{4}} \quad \text { with } \quad l\left(Q_{0}\right)=l_{0}
$$


Finally we reduce to the following differential problem:

$$
\left\{\begin{array}{l}
\eta l_{, Q} \dot{Q}=\left(\omega-\left(\frac{C}{2 \varepsilon L l(Q)}\right)^{\frac{1}{3}} Q^{\frac{4}{3}}\right)_{+} \\
l\left(Q_{0}\right)=l_{0}
\end{array}\right.
$$

whose solution is represented in Fig. 5 for the following values of the constants: $C=0.0014 \mathrm{MPa}^{-1}, L=210 \mathrm{~mm}, \varepsilon=10^{-2} L, l_{0}=0.05 \mathrm{~mm}, \eta=$ $1.7 e^{9} \mathrm{MPa} \mathrm{s}, \omega=-3.6 e^{6} \mathrm{~N} / \mathrm{mm}$ and $\dot{Q}=1 \mathrm{MPa} \mathrm{s}^{-1}$.

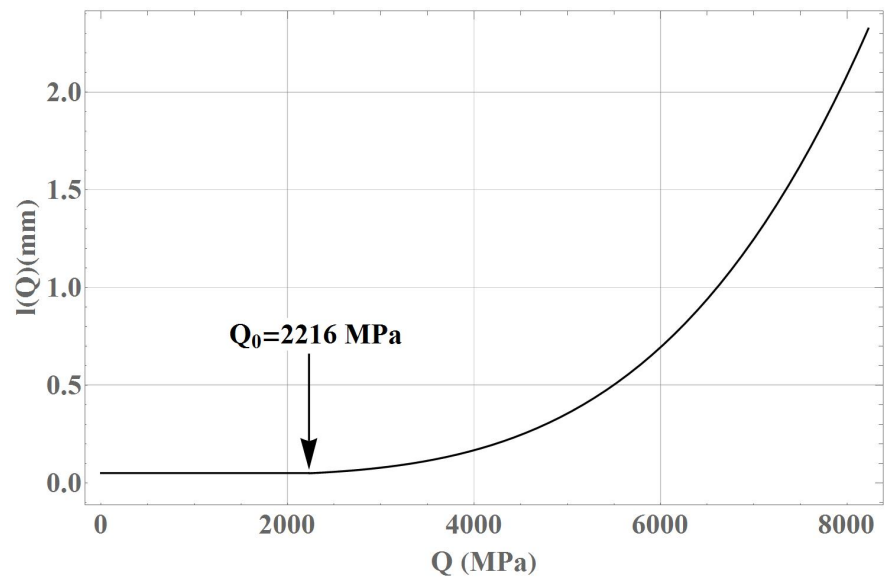

Fig. 5 Evolution of the damage variable $l(Q)$ as a function of the load $Q$, obtained as the solution of the differential problem (47).

Moreover, the displacement jump $\left[u_{3}\right]$, shown in Fig. 6, is expressed as follows:

$$
\left\{\begin{array}{l}
{\left[u_{3}\right]=\varepsilon^{\frac{2}{3}} Q^{\frac{1}{3}\left(\frac{4 C l_{0}^{2}}{L}\right)^{\frac{1}{3}}} \quad 0 \leq Q \leq Q_{0}} \\
{\left[u_{3}\right]=\varepsilon^{\frac{2}{3}} Q^{\frac{1}{3}\left(\frac{4 C l(Q)^{2}}{L}\right)^{\frac{1}{3}}} \quad Q>Q_{0}}
\end{array}\right.
$$

with $l(Q)$ solution of the differential problem (47).

\subsubsection{Mode II: simple shear condition}

In the reference configuration, the composite structure is subjected to a pure shear load $q>0$ directed along $i_{1}$ and acting on the upper and the lower boundaries $\Gamma_{+}$and $\Gamma_{-}$, as shown in Fig. 4. On the remaining part of the boundary $\partial \Omega^{0}$, the surface forces are taken to vanish. The load intensity $q$ is assumed to not depend on $\varepsilon$ and the body forces are neglected. In analogy with the previous case, the adherents are supposed to comprise the same Saint 


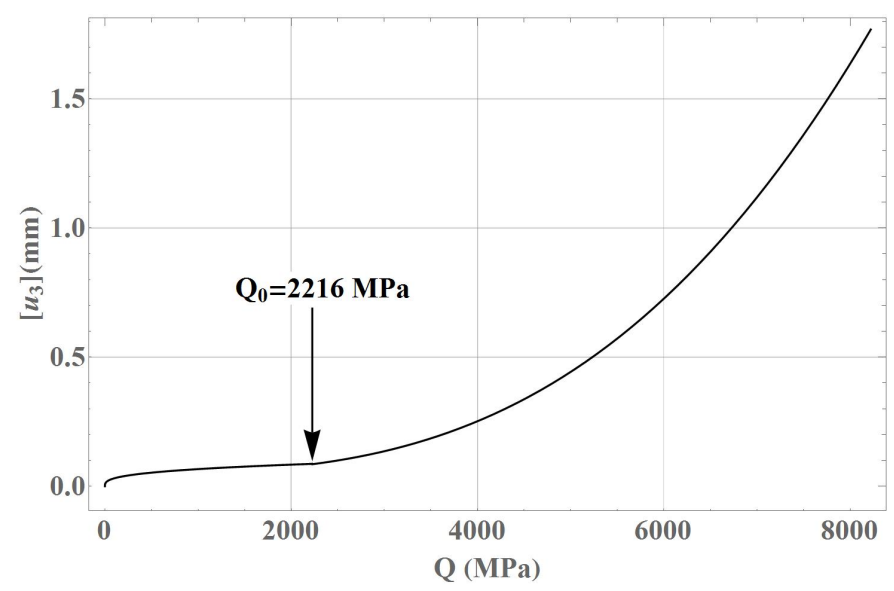

Fig. 6 Evolution of the displacement jump [us] as a function of the load $Q$.

Venant-Kirchhoff material and their engineering elastic constants $E, G$ and $\nu$ are used in what follows.

The equilibrium problem of the composite structure is analogous to the system (37), except for replacing the boundary condition on $\Gamma_{ \pm}$with

$$
P i_{3}=q i_{1} \text {. }
$$

We seek the following solution for the displacement field $u: \Omega^{0} \rightarrow \mathbb{R}^{3}$ :

$$
u=\left(\gamma x_{3} \pm \frac{1}{2}\left[u_{1}\right]\right) i_{1} \quad \text { in } \Omega_{ \pm}^{0}
$$

with $\gamma$ a constant to be determined, representing the shear strain in the plane $\left\{x_{1} x_{3}\right\}$ and $\left[u_{1}\right]$ the $i_{1}$-component of the displacement-jump vector $[u]$ at the interface $S^{0}$.

The first Piola-Kirchhoff stress tensor corresponding to (50) is:

$$
P=P_{11}\left(i_{1} \otimes i_{1}\right)+P_{22}\left(i_{2} \otimes i_{2}\right)+P_{33}\left(i_{3} \otimes i_{3}\right)+P_{13}\left(i_{1} \otimes i_{3}\right)+P_{31}\left(i_{3} \otimes i_{1}\right)
$$

with

$$
\begin{gathered}
P_{11}=\frac{E \gamma^{2}}{4(1+\nu)(1-2 \nu)}, \\
P_{22}=\frac{E \nu \gamma^{2}}{2(1+\nu)(1-2 \nu)}, \\
P_{33}=\frac{E(1-\nu) \gamma^{2}}{2(1+\nu)(1-2 \nu)}, \\
P_{13}=\frac{E}{2(1+\nu)(1-2 \nu)}\left(\gamma\left(\frac{1-2 \nu}{2}\right)+\gamma^{3}(1-\nu)\right), \\
P_{31}=\frac{E \gamma}{4(1+\nu)} .
\end{gathered}
$$


If the boundary condition (49) is imposed, then the following equation which determines $\gamma$ as a function of the load $q$ is derived:

$$
q=\gamma G\left(\gamma^{2} \frac{1-\nu}{1-2 \nu}+\frac{1}{2}\right)
$$

being $G=\frac{E}{2(1+\nu)}$ the shear modulus.

In analogy with the previous example, the following relationship holds:

$$
\left[u_{1}\right]=\varepsilon^{\frac{2}{3}} \frac{q}{|q|^{\frac{2}{3}}}\left(\frac{2}{b_{3333}(l)}\right)^{\frac{1}{3}}
$$

which can be rewritten as follows:

$$
\left\{\begin{array}{lc}
{\left[u_{1}\right]=\varepsilon^{\frac{2}{3}} q^{\frac{1}{3}}\left(\frac{4 C l_{0}^{2}}{L}\right)^{\frac{1}{3}}} & 0 \leq q \leq q_{0}, \\
{\left[u_{1}\right]=\varepsilon^{\frac{2}{3}} q^{\frac{1}{3}}\left(\frac{4 C l(q)^{2}}{L}\right)^{\frac{1}{3}}} & q>q_{0},
\end{array}\right.
$$

with $l(q)$ solution of the differential problem:

$$
\left\{\begin{array}{l}
\eta l_{, q} \dot{q}=\left(\omega-\left(\frac{C}{2 \varepsilon L l(q)}\right)^{\frac{1}{3}} q^{\frac{4}{3}}\right)_{+} \\
l\left(q_{0}\right)=l_{0}
\end{array}\right.
$$

in which $q_{0}=-\omega^{\frac{4}{3}}\left(\frac{2 \varepsilon L l_{0}}{C}\right)^{\frac{1}{4}}$.

From a qualitative point of view, the damage parameter $l(q)$ and the displacement jump $\left[u_{1}\right]$ show a behavior similar to the one shown in Figs. 5 and 6.

4.2 Validation at the macroscale: Vermeltfoort and Raijmakers walls

The proposed numerical simulations made reference to the extensive experimental campaign carried out in the nineties by Vermeltfoort and Raijmakers $[48,49]$ concerning shear tests on masonry walls. Vermeltfoort and Raijmakers results are well-known in structural analysis of masonry structures and they are usually taken as benchmarks for validating models [25, 1,51].

Experimental tests $[48,49]$ concern two one-meter-square and $100 \mathrm{~mm}$ thick walls, either without and with an opening, as sketched in Fig. 7. The central opening is one brick wide and six layers high and it is situated five layers from the bottom and one and half brick lengths from the loaded side of the specimen (see Fig. 7, left side). The walls comprise of full bricks of $210 * 100 * 52 \mathrm{~mm}^{3}$ and head and bed mortar joints of $10 \mathrm{~mm}$ thickness. The up and the bottom sides of the specimens are connected via a high-performance mortar with two steel beams. In particular, the beam at the bottom side is connected to the 
test ring. Moreover, the up side beam is prevented to move in vertical direction by three vertical jacks in displacement controlled mode, and it is forced to move horizontally by a jack placed on the left side of the stiff beam. The horizontal load acts at the upper edge of the specimen as an uniform distributed shear load [48]. Vermeltfoort and Raijmakers tested many specimens comprising units and mortars of different material properties, nevertheless, only the $J D$-type walls [25] are considered in the proposed numerical simulations, accordingly bricks have a Young modulus $E_{b}=16.7 \mathrm{GPa}$ and a Poisson ratio $\nu_{b}=0.15$ and mortar has a Young modulus $E_{m}=0.782 \mathrm{GPa}$ and a Poisson ratio $\nu_{m}=0.13$.
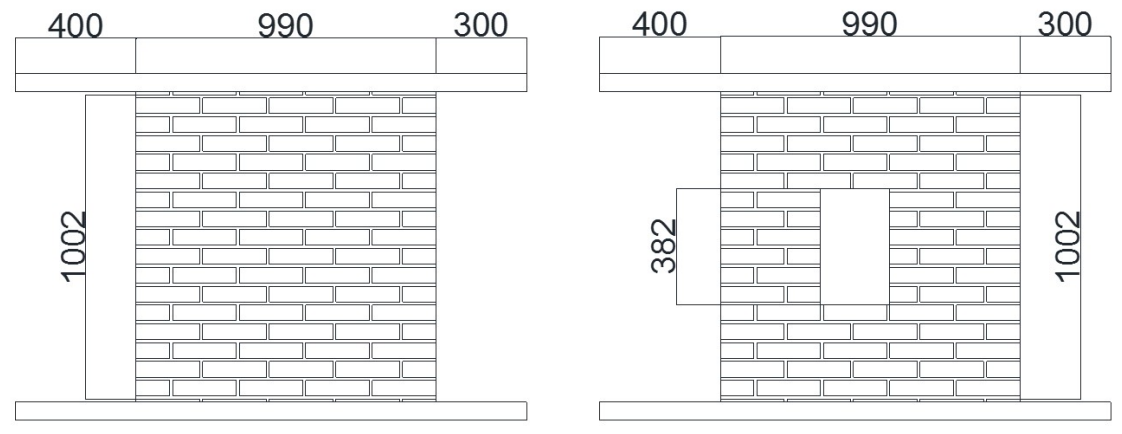

Fig. 7 Geometry of the walls used in experimental tests by Vermeltfoort and Raijmakers.

Both specimens (with and without opening) are subjected to a vertical preload of $0.3 \mathrm{MPa}$, then the shear force is imposed increasingly and monotonically via the horizontal jack. Concerning the wall without opening, some horizontal cracks caused by traction stresses and located at the application corner of the force and at its opposite in the bottom side, propagate rapidly when the horizontal load is applied. Successively, cracks propagate following the mortar joints path along the compressed diagonal with the load increasing, until the rupture. Moreover, the application of the horizontal load to the wall with opening causes first cracks nucleation and propagation localized at the corner of the central opening. Consecutively, after load increasing, a predominant diagonal crack is formed along the compressed diagonal. Finally, the rupture mechanism consists in some localized compressive failures at the top and at the bottom of the wall.

\subsubsection{Numerical modeling of the walls}

The proposed numerical simulations have been carried out with the FEMbased software Comsol Multiphysics. The vertical pre-loading is not taken into account for this study, accordingly, only the applied shear force is simulated. The problem is modeled in $2 \mathrm{D}$ plane stress conditions. The same boundary 
conditions are assumed for both walls, as sketched in Fig. 8. In particular, the bottom side beam is assumed to be fixed to a rigid plane. Moreover, the top side beam is constrained in the vertical direction and a horizontal displacement is applied on its left side. The simulations comprise a quasi-static loading in displacement controlled mode, accordingly the applied horizontal displacement is linearly increased until its maximal value.

The three principal domains, i. e. bricks, mortar and steel beams, are modeled as linear elastic isotropic materials whose material properties are taken in according with experiments by Vermeltfoort and Raijmakers [48,49], as detailed in the previous paragraph.

It is worth noting that the proposed finite element analyses are carried out within the small strain theory framework. This fact is not incompatible with the implementation of an interface law obtained via the finite strain hypothesis, as demonstrated in a previous work by authors [35].

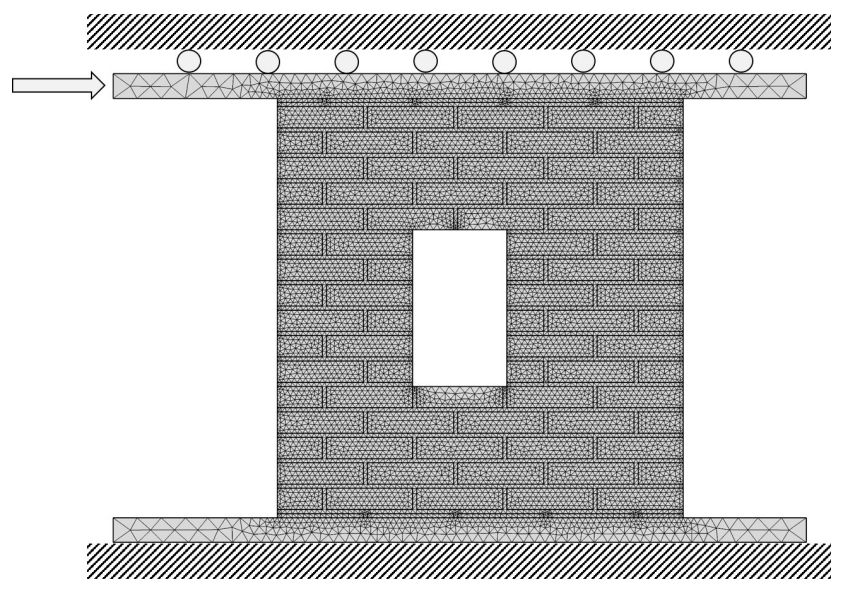

Fig. 8 Schematic sketch of mesh and boundary conditions for the wall with opening

A free triangular mesh of quadratic Lagrangian order is used in whole domains of both models, steel beams included (Fig. 8). The simulations are solved within a quasi-static framework via a temporal discrete solver included in Comsol. This choice has been made in order to limit solution convergence problems, which should be due to the strong nonlinearities of coupling brick/mortar interface laws with the evolution law of $l$, sixth and seventh equations of system (36), respectively. Consecutively, a fixed time step is chosen as $\Delta t=0.1 \mathrm{~s}$ and the shear test simulations are carried out within a range varying between 0 and $5 \mathrm{~s}$. Accordingly, the imposed displacement increases linearly during this time range and it varies between 0 at the initial step and its maximal value $d_{\max }=5.5 \mathrm{~mm}$ at the final step. 


\subsubsection{Brick/mortar interfaces modeling}

Within the present study the brick/mortar interfaces, either in horizontal and vertical principal directions of masonry walls, are assumed to be adhesive interfaces characterized by a nonlinear damaging behavior. These interfaces are governed, as a recall, by the following equations:

$$
\begin{cases}P_{i 3}=\frac{1}{2 \varepsilon^{2}} b_{3333}(l)|[u]|^{2}\left[u_{i}\right] & \text { on } S \\ \eta \dot{l}=\left(\omega-\frac{1}{8 \varepsilon^{3}} b_{3333, l}(l)|[u]|^{4}\right)_{+} & \text {on } S\end{cases}
$$

where the first equation represents the soft nonlinear interface law and the second one the nonlinear evolution of the damage parameter $l$. These equations are specialized in the case of horizontal and vertical interfaces. The interface stiffness $b_{3333}(l)$ is obtained via a micromechanical homogenization of Kachanov-type $[29,47,37,38,10]$, its closed-form expression reads as:

$$
b_{3333}\left(l_{j}\right)=\frac{L_{j}}{2 C l_{j}^{2}}
$$

where the subscript $j=h, v$ indicates the horizontal and vertical interface cases, respectively. The compliance $C$ is calculated from Eq. (2) starting from the undamaged interphase properties, and in the proposed models it results in $C=0.0014 \mathrm{MPa}^{-1}$ (refers to $[37,10]$ for further details). Parameter $L$ is the characteristic length of the interfaces, resulting in $L_{h}=210 \mathrm{~mm}$ and $L_{v}=52$ $\mathrm{mm}$ for horizontal and vertical interfaces, respectively. The first derivative of Eq. (62) with respect the damage parameter $l$ results in:

$$
b_{3333, l}\left(l_{j}\right)=-\frac{L_{j}}{C l_{j}^{3}}
$$

The interphase thickness $\varepsilon$ appearing in Eqs. (61) is specialized for horizontal and vertical interfaces and it is assumed to be a fraction of the characteristic length $L$, as follows: $\varepsilon_{h}=10^{-2} L_{h}, \varepsilon_{v}=10^{-2} L_{v}$. This last choice has been made in according with [19] in order to assure a good approximation of the interphase modeling problem with the physically-based interface one.

Let indicate $l\left(t^{k}\right)=l^{k}$ and $\left[u\left(t^{k}\right)\right]=\left[u^{k}\right]$ with $t^{k}=k \Delta t$, the damage parameter and the displacement jump vector calculated at the $k$-ieme time step, respectively. Similarly to the numerical approach in [32], by approximating the first derivative of the damage parameter with respect to time as $i \approx \frac{l^{k+1}-l^{k}}{\Delta t}$, an explicit form of the damaging equation (second equation of Eqs. (61)) is derived:

$$
l_{j}^{k+1}=l_{j}^{k}+\frac{\Delta t}{\eta}\left(\omega+\frac{L_{j}}{8 C \varepsilon_{j}^{3}\left(l_{j}^{k}\right)^{3}}\left|\left[u_{j}^{k}\right]\right|^{4}\right)_{+} \quad j=h, v
$$

Eq. (64), where Eq. (63) has been taken into account, is used for the numerical implementation of damaging in both horizontal and vertical interfaces of 
masonry walls. Moreover, parameter $l$ has as an inferior limit bound, its initial value $l^{0}$, so the following condition holds: $l^{k} \geq l^{0}=l(0)$. Model parameters $l^{0}=0.05 \mathrm{~mm}, \eta=1.7 e^{9}$ and $\omega=-3 e^{6}$ have been identified from experimental data by $[48,49]$ concerning solid walls of $J D$-type, and they are used in both proposed simulations, i. e. solid wall and wall with a central opening. As a result, the evolution of damage parameter $l_{h}$ in the solid wall is shown in Fig. 9.

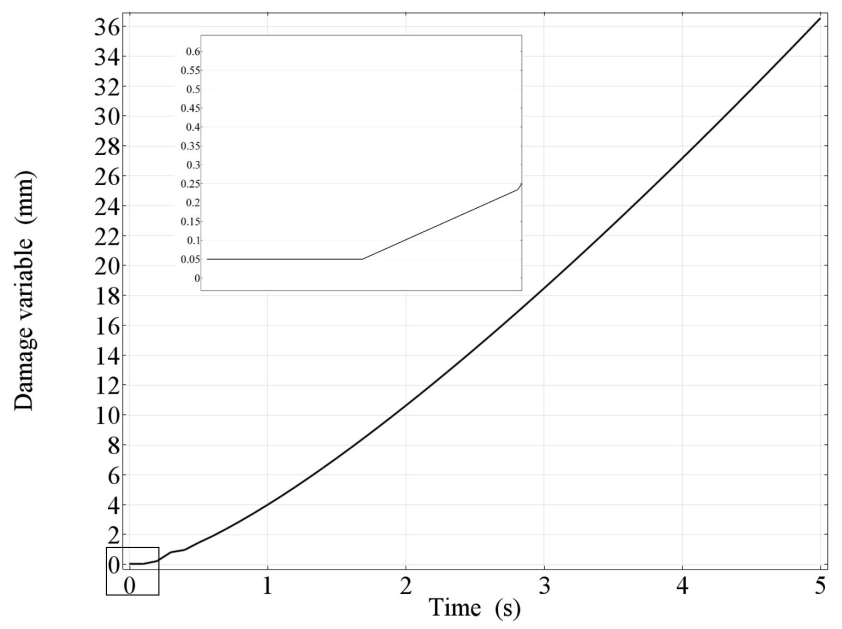

Fig. 9 Damaging in horizontal interfaces of the solid wall

The $l$ curve (Fig. 9) highlights a first path in which $l$ is constant and equal to the initial value $l^{0}$, then while load increases the damaging phenomenon begins. It is worth to remark that for the proposed numerical investigations a superior limit bound for the damage parameters is not taken into account, nevertheless it may be easily implemented. In the following section main results are discussed.

\subsubsection{Results and discussions}

Concerning the solid wall an identification process of the three model parameters $l^{0}, \eta$ and $\omega$ has been made. The obtained values allow to compare the global behavior of the simulated shear test with the experimental one $[48,49]$. Particularly, Fig. 10 shows the horizontal imposed force against the horizontal displacement of the top side of the wall, either in numerical and experimental case. A good correlation is found, specially after the elastic limit. It is worth to remark that the fracture phenomenon is not object of the present study, which focuses on damaging and the consequent loss of global stiffness.

The initial damage parameter $l^{0}=0.05 \mathrm{~mm}$ is assumed to be uniform in whole masonry wall at the initial step, this means assuming to have a small 
initial damage uniformly distributed at the brick/mortar interfaces, either horizontal and vertical. This pre-damaging can be caused, in the masonry manufacturing, by creep and shrinkage phenomena in the mortar. The parameter $l^{0}$ influences the initial elastic behavior of the wall.

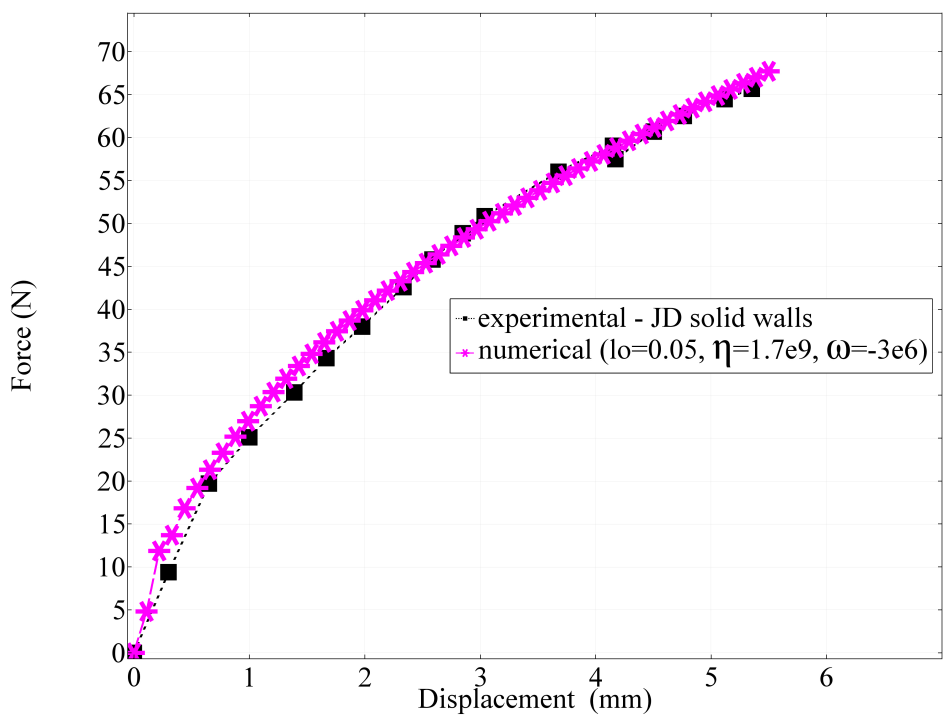

Fig. 10 Horizontal force-displacement graphs for solid wall. Comparison between the proposed model and the experimental data by [48,49] (black dotted curve)

The model parameters $\eta=1.7 e^{9}$ and $\omega=-3 e^{6}$ govern the post-peak masonry behavior, i. e. after the elastic limit, by acting on the damage evolution (Eqs. (61)). Particularly, $\omega$ represents an energy threshold beyond which damage triggers.

The distributions of $\sigma_{y y}$ stresses shown in Fig. 11 are in agreement with the experimental findings by Vermeltfoort and Raijmakers $[48,49]$. In details, these contour plots put in evidence, for solid wall as well as for the wall with the opening, the compressive and tensile zones at the top and bottom wall corner, and at the corner of the central opening. These stresses localization zones are the zones where experimentally cracks nucleate and begin propagating following the brick/mortar interfaces and/or the mortar joints. Within the numerical simulations, this last phenomenon is highlighted in Figs. 12 and 13, where the distribution of damage parameters $l_{h}$ and $l_{v}$ is shown.

Particularly, highest values of damage are localized, accordingly with the experimental evidences, at the corner of the wall in the solid wall, and at the opening corner in the wall with the opening. The latter finding confirms that the proposed interface model is able to make a good prediction of damaging in masonry structures. Therefore, for both numerical models, parameter $l_{v}$ is smaller than $l_{h}$. This result is related to the loading condition. In fact, by applying a shear loading in the horizontal direction the displacement jumps 
$\sigma_{y y}(\mathrm{MPa}) \mathrm{t}=5 \mathrm{~s}$

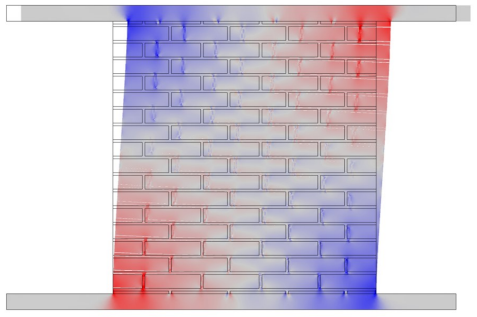

$\sigma_{\text {yy }}(\mathrm{MPa}) \mathrm{t}=5 \mathrm{~s}$

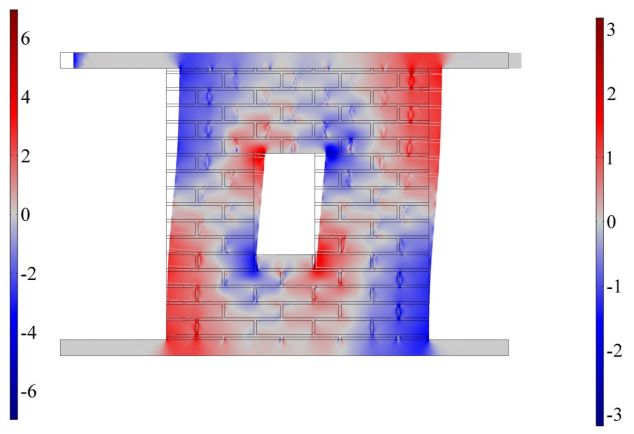

Fig. 11 Contour plots of yy-component of stress tensor with deformed shape at the final simulation step. Undeformed shape is traced also

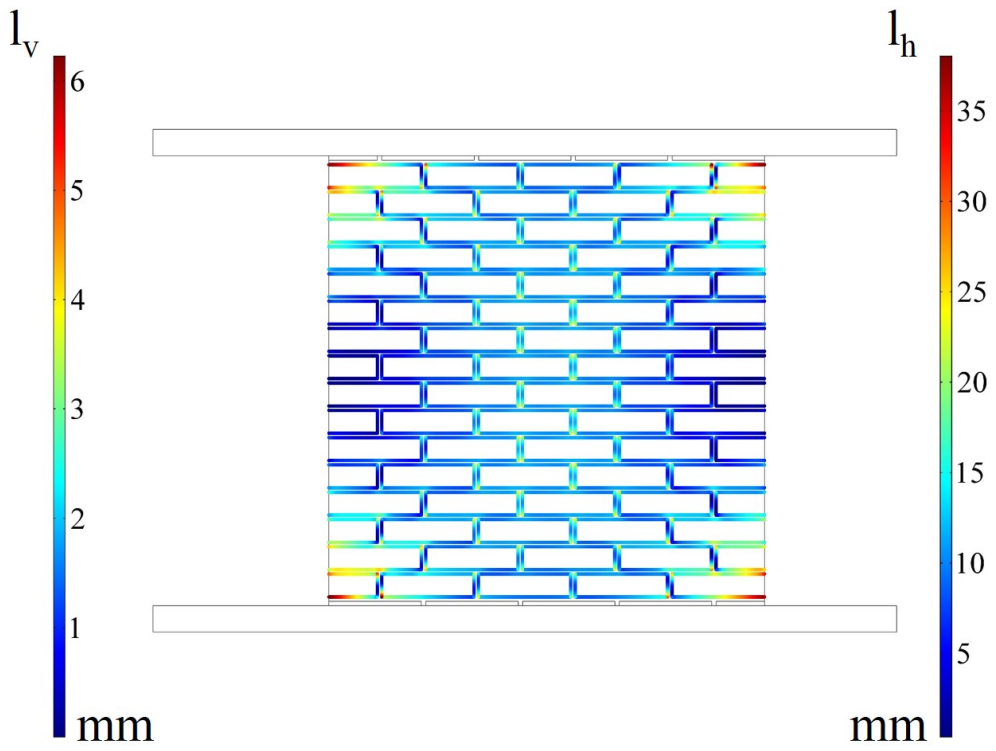

Fig. 12 Contour plots of the distribution of damage parameters $l_{h}$ and $l_{v}$ in solid wall

in horizontal interfaces are higher than in the vertical interfaces, providing higher concentrations of damage (see Eqs. 61).

\section{Conclusions}

In the present study a model of soft imperfect interface comprising large displacements and evolving microcracking is derived via asymptotic techniques. 


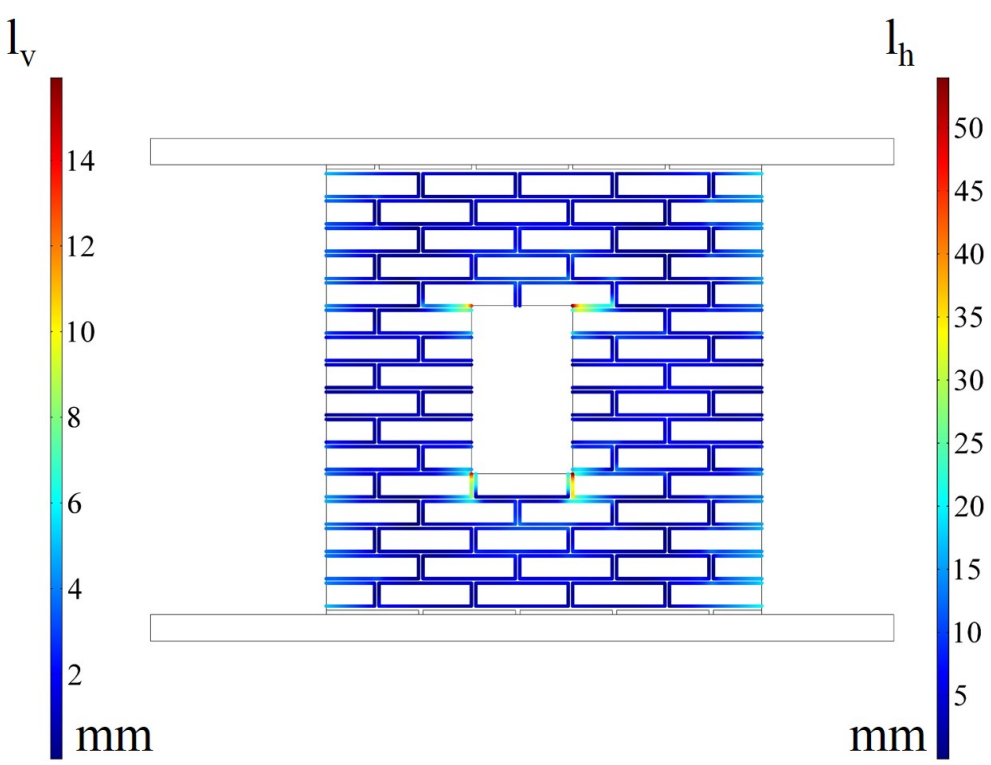

Fig. 13 Contour plots of the distribution of damage parameters $l_{h}$ and $l_{v}$ in wall with opening

This soft nonlinear interface is applied to describe damaging localized at the brick/mortar interfaces in masonry structures.

Theoretical results are supported by numerical and analytical validations. At the macroscale scale, two numerical examples based on well-established experimental data of masonry walls in shear loading condition by $[48,49]$ have been proposed. Numerical results show a good correlation with experiments in terms of the global behavior. Particularly, the predictive capacity of the proposed model to individuate the critical areas in which damaging occurs is highlighted.

The proposed interface model does not depend on loading conditions, on geometry and it is not mesh-dependent. Modeling the brick/mortar interfaces via the proposed imperfect interface model is a suitable and less computationally expensive alternative to existing detailed micro-models aimed at describing damage in masonry structures. Moreover, it allows to use linear elastic constitutive laws for the masonry's principal constituents, i.e., bricks and mortar joints.

A limitation of the proposed study is to not make a distinction between the damage level of compressive and tensile zones, as also highlighted in Figs. 12 and 13. As a future enhancement, an unilateral condition preventing damaging in compression will be accounted within the pseudo-potential of dissipation in order to better describe the general behavior of quasi-brittle materials. 
Acknowledgements This research was partially supported by Vinci Program 2013 (n. C2-73) of Italo-Francese University.

Conflict of interest The authors declare that they have no conflicts of interest.

\section{References}

1. Alfano G., Sacco E. (2006) Combining interface damage and friction in a cohesive-zone model, Int. J. Numer. Meth. Engng. 68 (5):542-582

2. Anthoine A. (1999) Derivation of the in-plane elastic characteristics of masonry through homogenization theory, Int. J. Solid Struct. 32 (2):137-163

3. Benveniste, Y. and Miloh, T. (2001) Imperfect soft and stiff interfaces in two-dimensional elasticity, Mechanics of Materials, 33 (6):309-323

4. Bonetti E., Bonfanti G., Lebon F., Rizzoni, R. (2016) A model of imperfect interface with damage, Meccanica, 1-12

5. Bonetti E., Bonfanti G., Rossi R. (2014) Analysis of a temperature-dependent model for adhesive contact with friction, Physica D, 285:42-62

6. Bonetti E., Bonfanti G., Rossi R. (2015) Modeling via the internal energy balance and analysis of adhesive contact with friction in thermoviscoelasticity, Nonlinear Anal. Real World Appl., 22:473-507

7. Ciarlet P.G. (1988) Mathematical Elasticity. Volume I: Three-Dimensional Elasticity, North-Holland, Amsterdam

8. Dumont, S.,Lebon, F., Rizzoni, R. (2014) An asymptotic approach to the adhesion of thin stiff films, Mech. Res. Com., 58:24-35

9. Fileccia Scimeni, G., Giambanco, G., Spada, A. (2014) The interphase model applied to the analysis of masonry structures, Comput. Methods Appl. Mech. Engrg. 279:6685

10. Fouchal, F., Lebon, F., Raffa, M.L., Vairo, G. (2014) An interface model including cracks and roughness applied to masonry, The Open Civil Engineering Journal, 8 (1):263-271

11. Fouchal F., Lebon F., Titeux, I. (2009) Contribution to the modelling of interfaces in masonry construction, Const. Build. Mat., 23:2428-2441

12. Frémond M. (2001) Non-Smooth Thermo-mechanics, Springer Eds

13. Gambarotta L., Lagomarsino S. (1997) Damage models for the seismic response of brick masonry shear walls. Part I: The mortar joint model and its applications, Earthquake Eng. Struct., 26 (4):423-439

14. Geymonat, G., Krasucki, F., Lenci, S. (1999) Mathematical analysis of a bonded joint with a soft thin adhesive, Math. Mech. Solid, 4 (2):201-225

15. Giambanco, G., Rizzo, S., Spallino, R. (2001) Numerical analysis of masonry structures via interface models, Comput. Methods Appl. Mech. Engrg. 190:64936511

16. Giambanco, G., Fileccia Scimeni, G., Spada, A. (2012) The interphase finite element, Comput. Mech. 50:353366

17. Hashin, Z. (2002) Thin interphase/imperfect interface in elasticity with application to coated fiber composites, J. Mech. Phys. Solid, 50 (12):2509-2537

18. Klarbring A. (1991) Derivation of the adhesively bonded joints by the asymptotic expansion method, Int. J. Engng. Sci., 29:493-512

19. Lebon, F., Ould Khaoua, A., Licht, C. (1998) Numerical study of soft adhesively bonded joints in finite elasticity, Comp. Mech., 21:134-140

20. Lebon, F., Rizzoni, R. (2008) Asymptotic study on a soft thin layer: The non-convex case, Mech. Adv. Mat. Struct., 15:12-20

21. Lebon, F., Rizzoni, R. (2010) Asymptotic analysis of a thin interface: The case involving similar rigidity, Int. J. Engng. Sci., 48:473-486

22. Lebon, F., Rizzoni, R. (2011) Asymptotic behavior of a hard thin linear elastic interphase: An energy approach, Int. J. Solid Struct., 48:441-449

23. Lebon, F., Ronel-Idrissi, S. (2004) Asymptotic analysis of Drucker-Prager and MohrCoulomb soft thin interfaces, Steel Comp. Struct., 4:133-147

24. Lebon, F., Zaittouni, S. (2010) Asymptotic modelling of interfaces taking contact conditions into account: Asymptotic expansions and numerical implementation, Int. J. Engn. Sci., 48:111-127 
25. Lourenço, P. (1996) Computational strategies for masonry structures, PhD thesis, Faculdade de engenharia da Universidade do Porto

26. Lourenço P.B., Rots J.G. (1997) Multisurface interface model for analysis of masonry structures, J. Eng. Mech., 123 (7):660-668

27. Lourenço P.B., Rots J.G., Blaauwendraad J. (1998) Continuum model for masonry: Parameter estimation and validation, J. Struct. Eng., 124 (6):642-652

28. Lotfi H.R., Shing, P.B. (1994) Interface model applied to fracture of masonry structures, J. Struct. Eng., 120 (1):63-80

29. Mauge C., Kachanov M. (1994) Effective elastic properties of an anisotropic material with arbitrarily oriented interacting cracks, J. Mech. Phys. Solid, 42:561-584

30. Medeiros P., Vasconcelos G., Loureno P.B., Gouveia J. (2013) Numerical modelling of non-confined and confined masonry walls, Constr. Build. Mater., 41:968-976

31. Milani G., Loureno P.B., Tralli A. (2006) Homogenised limit analysis of masonry walls, part I: failure surfaces, Comput. Struct., 84 (3-4):166-180

32. Orefice, A., Mancusi G., Lebon, F., Dumont S. (2016) An experimental/numerical study on the interfacial damage of bonded joints for Fibre-Reinforced Polymer profiles at service conditions, Technologies 4(3):20

33. Pelissou, C., Lebon, F. (2009) Asymptotic modeling of quasi-brittle interfaces, Comput. Struct., 87:1216-1223

34. Raffa, M.L. (2015) Micromechanical modeling of imperfect interfaces and applications, $\mathrm{PhD}$ thesis, University of Rome Tor Vergata and Aix-Marseille University.

35. Raffa, M.L., Lebon, F., Rizzoni R. (2016) On modelling brick/mortar interface via a St. Venant-Kirchhoff orthotropic soft interface. Part I: theory, Int. J. Masonry Research and Innovation, 1 (2):142-164

36. Raffa, M.L., Lebon, F., Rizzoni R. (2016) On modelling brick/mortar interface via a St. Venant-Kirchhoff orthotropic soft interface. Part II: in silico analysis, Int. J. Masonry Research and Innovation, to appear.

37. Rekik A., Lebon, F. (2010) Identification of the representative crack length evolution in a multi-level interface model for quasi-brittle masonry, Int. J. Solid Struct., 47 (2223):3011-3021

38. Rekik A., Lebon, F. (2012) Homogenization methods for interface modeling in damaged masonry, Adv. Eng. Software, 46 (1):35-42

39. Rizzoni, R., Lebon, F. (2012) Asymptotic analysis of an adhesive joint with mismatch strain, Eur. J. Mech. A/Sol., 36:1-8

40. Rizzoni, R., Lebon, F. (2013) Imperfect interfaces as asymptotic models of thin curved elastic adhesive interphases, Mech. Res. Comm., 51: 39-50

41. Rizzoni, R., Dumont, S., Lebon, F. (2017) On Saint Venant - Kirchhoff Imperfect Interfaces Int. J. Nonlinear Mech. 89:101-115

42. Rizzoni, R., Dumont, S., Lebon, F., Sacco, S. (2014) Higher order model for soft and hard interfaces, Int. J. Solid Struct., 51:4137-4148

43. Rots J.G. (1991) Numerical simulation of cracking in structural masonry, Heron, 36 (2):49-63

44. Sacco E. (2009) A nonlinear homogenization procedure for periodic masonry, Eur. J. Mech. A Solids 28 (2):209-222

45. Serpilli, M. (2014) Asymptotic analysis of a multimaterial with a thin piezoelectric interphase, Meccanica, 49 (7):1641-1652

46. Sevostianov, I., Kachanov, M. (2014) On some controversial issues in effective field approaches to the problem of the overall elastic properties, Mechanics of Materials, 69:93105

47. Tsukrov, I., Kachanov, M. (2000) Effective moduli of an anisotropic material with elliptical holes of arbitrary orientational distribution, Int. J. Solid Struct., 37 (41):5919-5941

48. Vermeltfoort, A. T., Raijmakers, T. M. J (1992) Shear tests on masonry panels of $1 x 1$ m2, in A. T. Vermeltfoort (Ed.), Research on building structures and building physics : proceedings of an interuniversity research seminar, Eindhoven: University of Technology, 171-177

49. Vermeltfoort, A. T., Raymakers, T. M. J., Janssen, H. J. M. (1993) Shear tests on masonry walls, in A. A. Hamid, H. G. Harris (Eds.), 6th North American Masonry Conference, 6-9 June 1993, Philadelphia, Pennsylvania, USA, Lancaster: Technomic Publ. Co., $1183-1193$ 
50. Zucchini A., Lourenço P.B (2002) A micro-mechanical model for the homogenisation of masonry, Int. J. Solid Struct. 39 (12):3233-3255

51. Zucchini A., Lourenço P.B (2009) A micro-mechanical homogenisation model for masonry: Application to shear walls, Int. J. Solid Struct. 46 (3-4):871-886 